Supporting Information of

\title{
Analytic energy gradients and Hessians of exact two-component relativistic methods: efficient implementation and applications
}

Wenli Zou, Guina Guo, Bingbing Suo, and Wenjian Liu

Table S1: Harmonic vibrational frequencies (in $\mathrm{cm}^{-1}$ ) of $\mathrm{Pt}_{2} \mathrm{Rn}_{2} \mathrm{~F}_{4}$. Values in parentheses are frequencies of the identity and commutation approximations ( $c f$. Eqs. (42) and (41) in the text), respectively.

\begin{tabular}{lrrr}
\hline \hline & sf-X2C & sf-X2C/AXR & sf-X2C/AU \\
\hline$\omega\left(1 b_{3 u}\right)$ & $24.6(23.9,23.5)$ & $24.7(23.7,23.5)$ & 21.5 \\
$\omega\left(1 a_{g}\right)$ & $52.1(52.3,52.0)$ & $52.4(52.3,52.4)$ & 51.7 \\
$\omega\left(1 b_{1 u}\right)$ & $92.0(91.4,91.3)$ & $91.9(91.4,91.3)$ & 90.4 \\
$\omega\left(1 b_{3 g}\right)$ & $106.4(106.0,106.2)$ & $106.3(105.9,106.1)$ & 105.7 \\
$\omega\left(1 a_{u}\right)$ & $110.7(110.4,109.7)$ & $110.6(110.2,109.7)$ & 107.6 \\
$\omega\left(1 b_{2 u}\right)$ & $110.9(110.4,110.7)$ & $110.8(110.3,110.6)$ & 110.3 \\
$\omega\left(2 a_{g}\right)$ & $111.6(111.4,111.5)$ & $111.6(111.4,111.6)$ & 111.0 \\
$\omega\left(1 b_{2 g}\right)$ & $115.6(115.4,114.8)$ & $115.6(115.3,114.8)$ & 113.0 \\
$\omega\left(1 b_{1 g}\right)$ & $127.1(126.9,126.2)$ & $127.0(126.5,126.2)$ & 124.1 \\
$\omega\left(2 b_{3 u}\right)$ & $131.9(131.7,131.1)$ & $131.8(131.5,131.0)$ & 129.1 \\
$\omega\left(2 b_{3 g}\right)$ & $141.2(140.6,140.2)$ & $141.1(140.4,140.1)$ & 138.7 \\
$\omega\left(2 b_{1 u}\right)$ & $150.2(149.6,149.3)$ & $150.1(149.5,149.3)$ & 148.0 \\
$\omega\left(2 b_{2 u}\right)$ & $211.8(211.5,211.1)$ & $211.7(211.4,211.1)$ & 210.2 \\
$\omega\left(3 a_{g}\right)$ & $215.0(214.8,214.4)$ & $214.9(214.6,214.3)$ & 213.5 \\
$\omega\left(3 b_{3 g}\right)$ & $597.8(597.9,597.9)$ & $597.8(597.9,598.0)$ & 598.0 \\
$\omega\left(3 b_{1 u}\right)$ & $604.2(604.4,604.3)$ & $604.2(604.3,604.4)$ & 604.4 \\
$\omega\left(3 b_{2 u}\right)$ & $626.2(626.4,626.4)$ & $626.2(626.3,626.5)$ & 626.4 \\
$\omega\left(4 a_{g}\right)$ & $629.5(629.8,629.7)$ & $629.5(629.7,629.9)$ & 629.8 \\
\hline
\end{tabular}


Cartesian coordinates of $\mathrm{Au}_{14}$ by sf-X2C/PBE0.

\begin{tabular}{|c|c|c|c|}
\hline $\mathrm{Au}$ & 0.000000 & 1.301287 & 3.455717 \\
\hline $\mathrm{Au}$ & 0.000000 & -1.301287 & 3.455717 \\
\hline $\mathrm{Au}$ & 0.000000 & -2.411924 & 1.122548 \\
\hline $\mathrm{Au}$ & 0.000000 & -3.442601 & -1.385463 \\
\hline $\mathrm{Au}$ & 0.000000 & -1.299933 & -3.054007 \\
\hline $\mathrm{Au}$ & 0.000000 & 1.299933 & -3.054007 \\
\hline $\mathrm{Au}$ & 0.000000 & 3.442601 & -1.385463 \\
\hline $\mathrm{Au}$ & 0.000000 & 2.411924 & 1.122548 \\
\hline $\mathrm{Au}$ & 1.289497 & 0.000000 & 1.495924 \\
\hline $\mathrm{Au}$ & 1.338260 & -1.268643 & -0.817359 \\
\hline $\mathrm{Au}$ & 1.338260 & 1.268643 & -0.817359 \\
\hline $\mathrm{Au}$ & -1.289497 & 0.000000 & 1.495924 \\
\hline $\mathrm{Au}$ & -1.338260 & -1.268643 & -0.817359 \\
\hline $\mathrm{Au}$ & -1.338260 & 1.268643 & -0.817359 \\
\hline
\end{tabular}

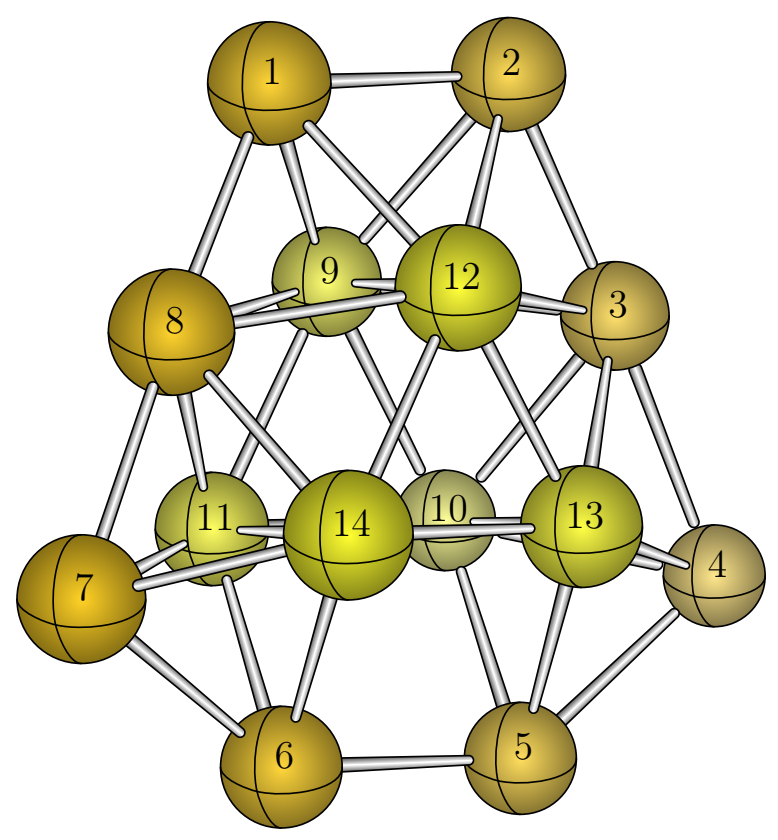

Figure S1: Geometry of $\mathrm{Au}_{14}$. Atoms in the two 3-membered rings are highlighted in light yellow. 
Table S2: Geometric parameters (in $\AA$ for bond lengths and degree for bond angles) and harmonic vibrational frequencies (in $\mathrm{cm}^{-1}$ ) of $\mathrm{Au}_{14}$. Values in parentheses are frequencies of the identity and commutation approximations ( $c f$. Eqs. (42) and (41) in the text), respectively.

\begin{tabular}{|c|c|c|c|}
\hline & sf-X2C & sf-X2C/AXR & $\mathrm{sf}-\mathrm{X} 2 \mathrm{C} / \mathrm{AU}$ \\
\hline$r_{14,13}=r_{11,10}$ & 2.537 & 2.537 & 2.537 \\
\hline$r_{12,9}$ & 2.579 & 2.579 & 2.578 \\
\hline$r_{1,8}=r_{2,3}$ & 2.584 & 2.584 & 2.584 \\
\hline$r_{6,5}$ & 2.600 & 2.600 & 2.599 \\
\hline$r_{1,2}$ & 2.603 & 2.603 & 2.602 \\
\hline$r_{14,6}=r_{11,6}=r_{13,5}=r_{10,5}$ & 2.607 & 2.607 & 2.606 \\
\hline$r_{14,7}=r_{11,7}=r_{13,4}=r_{10,4}$ & 2.615 & 2.615 & 2.614 \\
\hline$r_{8,14}=r_{8,11}=r_{3,13}=r_{3,10}$ & 2.619 & 2.619 & 2.618 \\
\hline$r_{12,14}=r_{12,13}=r_{9,11}=r_{9,10}$ & 2.639 & 2.639 & 2.638 \\
\hline$r_{14,11}=r_{13,10}$ & 2.677 & 2.677 & 2.675 \\
\hline$r_{1,12}=r_{1,9}=r_{2,12}=r_{2,9}$ & 2.683 & 2.683 & 2.682 \\
\hline$r_{8,7}=r_{3,4}$ & 2.712 & 2.712 & 2.711 \\
\hline$r_{7,6}=r_{4,5}$ & 2.716 & 2.716 & 2.715 \\
\hline$r_{12,8}=r_{12,3}=r_{9,8}=r_{9,3}$ & 2.760 & 2.760 & 2.760 \\
\hline$L_{12,8,9}=L_{12,3,9}$ & 55.7 & 55.7 & 55.7 \\
\hline$L_{1,12,8}=L_{1,9,8}=\angle_{2,12,3}=L_{2,9,3}$ & 56.7 & 56.7 & 56.7 \\
\hline$L_{12,1,9}=\angle 12,2,9$ & 57.5 & 57.5 & 57.4 \\
\hline$L_{14,12,13}=L_{11,9,10}$ & 57.5 & 57.5 & 57.5 \\
\hline$\angle_{8,12,14}=\angle_{3,12,13}=\angle_{8,9,11}=\angle_{3,9,10}$ & 58.0 & 58.0 & 58.0 \\
\hline$L_{1,12,2}=L_{1,9,2}$ & 58.0 & 58.0 & 58.0 \\
\hline$\angle_{14,7,6}=\angle_{11,7,6}=\angle_{13,4,5}=\angle_{10,4,5}$ & 58.5 & 58.5 & 58.5 \\
\hline$L_{12,8,14}=\angle_{12,3,13}=\angle_{9,8,11}=\angle_{9,3,10}$ & 58.7 & 58.7 & 58.7 \\
\hline$L_{14,8,7}=L_{11,8,7}=\angle_{13,3,4}=L_{10,3,4}$ & 58.7 & 58.7 & 58.7 \\
\hline$\angle_{14,6,7}=\angle_{11,6,7}=\angle_{13,5,4}=\angle_{10,5,4}$ & 58.8 & 58.8 & 58.8 \\
\hline$\angle_{8,7,14}=\angle_{8,7,11}=\angle_{3,4,13}=\angle_{3,4,10}$ & 58.9 & 58.9 & 58.9 \\
\hline$L_{11,14,6}=L_{14,11,6}=L_{10,13,5}=L_{13,10,5}$ & 59.1 & 59.1 & 59.1 \\
\hline$L_{11,14,7}=L_{14,11,7}=L_{10,13,4}=L_{13,10,4}$ & 59.2 & 59.2 & 59.2 \\
\hline$\angle_{8,14,11}=\angle 8,11,14=\angle 3,13,10=\angle 3,10,13$ & 59.3 & 59.3 & 59.3 \\
\hline$L_{1,8,12}=\angle_{1,8,9}=\angle_{2,3,12}=\angle_{2,3,9}$ & 60.2 & 60.2 & 60.2 \\
\hline$\angle_{2,1,12}=\angle_{2,1,9}=\angle_{1,2,12}=\angle_{1,2,9}$ & 61.0 & 61.0 & 61.0 \\
\hline$L_{12,14,13}=\angle_{12,13,14}=\angle_{9,11,10}=\angle 9,10,11$ & 61.3 & 61.3 & 61.3 \\
\hline$\angle_{1,12,9}=\angle_{1,9,12}=\angle_{2,12,9}=\angle_{2,9,12}$ & 61.3 & 61.3 & 61.3 \\
\hline$L_{14,8,11}=L_{13,3,10}$ & 61.4 & 61.4 & 61.4 \\
\hline$\angle_{14,7,11}=\angle_{13,4,10}$ & 61.6 & 61.6 & 61.5 \\
\hline$L_{14,6,11}=L_{13,5,10}$ & 61.8 & 61.8 & 61.8 \\
\hline$\angle 9,12,8=\angle 9,12,3=\angle 12,9,8=\angle_{12,9,3}$ & 62.2 & 62.2 & 62.2 \\
\hline$\angle_{8,14,7}=\angle_{8,11,7}=\angle \angle_{3,13,4}=\angle_{3,10,4}$ & 62.4 & 62.4 & 62.4 \\
\hline$\angle_{7,14,6}=\angle 7,11,6=\angle 4,13,5=\angle 4,10,5$ & 62.7 & 62.7 & 62.7 \\
\hline$\angle 12,1,8=\angle 9,1,8=\angle 12,2,3=\angle 9,2,3$ & 63.2 & 63.2 & 63.2 \\
\hline$\angle_{12,14,8}=\angle \angle_{12,13,3}=\angle 9,11,8=\angle 9,10,3$ & 63.3 & 63.3 & 63.3 \\
\hline$\angle_{12,8,11}=\angle \angle_{12,3,10}=\angle 9,8,14=\angle 9,3,13$ & 87.6 & 87.6 & 87.6 \\
\hline$\angle_{12,14,11}=\angle 12,13,10=\angle 9,11,14=\angle 9,10,13$ & 88.9 & 88.9 & 88.9 \\
\hline$L_{14,6,5}=L_{11,6,5}=L_{13,5,6}=\angle_{10,5,6}$ & 89.3 & 89.3 & 89.3 \\
\hline$\angle 11,14,13=\angle 14,11,10=\angle 14,13,10=\angle 11,10,13$ & 90.0 & 90.0 & 90.0 \\
\hline$L_{13,14,6}=\angle_{14,13,5}=\angle_{10,11,6}=\angle_{11,10,5}$ & 90.7 & 90.7 & 90.7 \\
\hline$\angle_{9,12,14}=\angle 9,12,13=\angle 12,9,11=\angle L_{12,9,10}$ & 91.1 & 91.1 & 91.1 \\
\hline$\angle_{8,7,6}=\angle_{3,4,5}$ & 105.6 & 105.6 & 105.6 \\
\hline$\angle_{1,12,3}=\angle L_{1,9,3}=\angle 2,12,8=\angle 2,9,8$ & 107.3 & 107.3 & 107.3 \\
\hline
\end{tabular}


Table S2: (continued)

\begin{tabular}{|c|c|c|c|}
\hline & sf-X2C & sf-X2C/AXR & sf-X2C/AU \\
\hline$\angle_{8,12,13}=\angle_{3,12,14}=\angle_{8,9,10}=\angle_{3,9,11}$ & 108.1 & 108.1 & 108.1 \\
\hline$\angle_{8,14,6}=\angle_{8,11,6}=\angle 3,13,5=\angle 3,10,5$ & 111.6 & 111.6 & 111.6 \\
\hline$\angle_{1,12,14}=\angle_{1,9,11}=\angle_{2,12,13}=\angle 2,9,10$ & 114.6 & 114.6 & 114.6 \\
\hline$L_{2,1,8}=L_{1,2,3}$ & 115.5 & 115.5 & 115.4 \\
\hline$\angle_{8,14,13}=\angle 8,11,10=\angle \angle_{3,13,14}=\angle 3,10,11$ & 115.9 & 115.9 & 115.9 \\
\hline$L_{12,8,7}=L_{12,3,4}=\angle_{9,8,7}=L_{9,3,4}$ & 117.2 & 117.2 & 117.2 \\
\hline$L_{1,8,14}=L_{1,8,11}=\angle 2,3,13=L_{2,3,10}$ & 118.8 & 118.8 & 118.8 \\
\hline$\angle_{8,12,3}=\angle 8,9,3$ & 121.8 & 121.8 & 121.8 \\
\hline$\angle_{12,14,7}=\angle_{12,13,4}=\angle{ }_{9,11,7}=\angle 9,10,4$ & 125.5 & 125.5 & 125.5 \\
\hline$L_{12,14,6}=\angle_{12,13,5}=\angle_{9,11,6}=\angle_{9,10,5}$ & 138.5 & 138.5 & 138.5 \\
\hline$L_{7,6,5}=L_{4,5,6}$ & 142.1 & 142.1 & 142.1 \\
\hline$L_{13,14,7}=\angle 14,13,4=L_{10,11,7}=L_{11,10,4}$ & 146.2 & 146.2 & 146.2 \\
\hline$L_{1,12,13}=\angle_{1,9,10}=\angle_{2,12,14}=\angle_{2,9,11}$ & 151.9 & 151.9 & 152.0 \\
\hline$L_{1,8,7}=\angle_{2,3,4}$ & 176.9 & 176.9 & 176.9 \\
\hline$\omega\left(1 a_{2}\right)$ & $37.3(37.5,37.3)$ & $37.3(37.4, \quad 37.3)$ & 37.7 \\
\hline$\omega\left(1 b_{2}\right)$ & $55.8(55.9,55.8)$ & $55.7(55.9,55.8)$ & 55.8 \\
\hline$\omega\left(2 a_{2}\right)$ & $57.9(58.0,57.9)$ & $57.9(57.9, \quad 57.9)$ & 57.2 \\
\hline$\omega\left(1 b_{1}\right)$ & $61.3(61.3,61.3)$ & $61.3(61.3,61.3)$ & 60.7 \\
\hline$\omega\left(3 a_{2}\right)$ & $71.8(71.9,71.8)$ & $71.8(71.9,71.8)$ & 72.4 \\
\hline$\omega\left(2 b_{1}\right)$ & $84.6(84.7,84.6)$ & $84.6(84.7,84.6)$ & 84.9 \\
\hline$\omega\left(2 b_{2}\right)$ & $86.0(86.0,86.0)$ & $86.0(86.0,86.0)$ & 85.4 \\
\hline$\omega\left(1 a_{1}\right)$ & $89.8(89.9,89.8)$ & $89.8(89.9, \quad 89.8)$ & 89.0 \\
\hline$\omega\left(3 b_{1}\right)$ & $92.0(92.1,92.0)$ & $92.0(92.1,92.0)$ & 90.9 \\
\hline$\omega\left(4 a_{2}\right)$ & $99.7(99.7,99.7)$ & $99.6(99.7,99.6)$ & 99.8 \\
\hline$\omega\left(2 a_{1}\right)$ & $100.3(100.4,100.3)$ & $100.3(100.4,100.3)$ & 99.5 \\
\hline$\omega\left(3 b_{2}\right)$ & $103.3(103.4,103.3)$ & $103.3(103.4,103.3)$ & 102.2 \\
\hline$\omega\left(4 b_{2}\right)$ & $113.7(113.8,113.7)$ & $113.7(113.7,113.7)$ & 114.9 \\
\hline$\omega\left(3 a_{1}\right)$ & $121.2(121.3,121.2)$ & $121.2(121.3,121.2)$ & 121.7 \\
\hline$\omega\left(4 b_{1}\right)$ & $123.4(123.5,123.4)$ & $123.4(123.5,123.4)$ & 124.3 \\
\hline$\omega\left(5 b_{2}\right)$ & $127.5(127.6,127.5)$ & $127.5(127.6,127.5)$ & 126.8 \\
\hline$\omega\left(5 a_{2}\right)$ & $137.2(137.4,137.2)$ & $137.2(137.3,137.2)$ & 138.1 \\
\hline$\omega\left(6 b_{2}\right)$ & $142.2(142.3,142.3)$ & $142.2(142.3,142.2)$ & 142.4 \\
\hline$\omega\left(4 a_{1}\right)$ & $144.1(144.1,144.1)$ & $144.0(144.1,144.1)$ & 144.8 \\
\hline$\omega\left(5 a_{1}\right)$ & $151.0(151.1,151.0)$ & $151.0(151.1,151.0)$ & 151.2 \\
\hline$\omega\left(5 b_{1}\right)$ & $154.2(154.3,154.2)$ & $154.1(154.3,154.2)$ & 154.9 \\
\hline$\omega\left(7 b_{2}\right)$ & $164.3(164.4,164.3)$ & $164.3(164.3,164.3)$ & 165.9 \\
\hline$\omega\left(6 a_{2}\right)$ & $165.6(165.8,165.6)$ & $165.6(165.7,165.6)$ & 166.4 \\
\hline$\omega\left(6 a_{1}\right)$ & $169.2(169.3,169.2)$ & $169.2(169.2,169.2)$ & 169.1 \\
\hline$\omega\left(7 a_{1}\right)$ & $179.7(179.7,179.7)$ & $179.7(179.7,179.7)$ & 178.8 \\
\hline$\omega\left(7 a_{2}\right)$ & $194.8(195.0,194.8)$ & $194.8(194.9,194.8)$ & 195.6 \\
\hline$\omega\left(8 a_{1}\right)$ & $199.4(199.5,199.4)$ & $199.4(199.5,199.4)$ & 200.9 \\
\hline$\omega\left(9 a_{1}\right)$ & $201.5(201.5,201.5)$ & $201.4(201.5,201.5)$ & 202.4 \\
\hline$\omega\left(6 b_{1}\right)$ & $203.0(203.2,203.1)$ & $203.0(203.1,203.0)$ & 203.8 \\
\hline$\omega\left(8 b_{2}\right)$ & $206.6(206.6,206.6)$ & $206.6(206.6,206.6)$ & 207.0 \\
\hline$\omega\left(9 b_{2}\right)$ & $221.9(221.9,221.9)$ & $221.8(221.9,221.9)$ & 222.4 \\
\hline$\omega\left(10 a_{1}\right)$ & $223.1(223.1,223.1)$ & $223.1(223.1,223.1)$ & 224.1 \\
\hline$\omega\left(7 b_{1}\right)$ & $241.8(241.9,241.8)$ & $241.8(241.9,241.8)$ & 243.5 \\
\hline$\omega\left(10 b_{2}\right)$ & $241.9(241.9,241.9)$ & $241.9(241.9,241.9)$ & 243.0 \\
\hline$\omega\left(11 a_{1}\right)$ & $242.8(242.8,242.8)$ & $242.8(242.8,242.8)$ & 243.6 \\
\hline$\omega\left(12 a_{1}\right)$ & $262.6(262.7,262.6)$ & $262.6(262.7,262.6)$ & 263.9 \\
\hline
\end{tabular}


Cartesian coordinates of $\mathrm{X}_{2}, \mathrm{HgX}_{2}, \mathrm{HgX}_{4}$, and transition states $(\mathrm{X}=\mathrm{F}, \mathrm{Cl}$, and $\mathrm{CN}$ ) by (sf-)X2C/PBE0.

\begin{tabular}{|c|c|c|c|c|c|c|c|}
\hline \multicolumn{4}{|c|}{ sf-X2C } & \multicolumn{4}{|l|}{$\mathrm{X} 2 \mathrm{C}$} \\
\hline \multicolumn{8}{|l|}{ F2 } \\
\hline $\mathrm{F}$ & 0.000000 & 0.000000 & -0.688383 & $\mathrm{~F}$ & 0.000000 & 0.000000 & -0.688391 \\
\hline $\mathrm{F}$ & 0.000000 & 0.000000 & 0.688383 & $\mathrm{~F}$ & 0.000000 & 0.000000 & 0.688391 \\
\hline \multicolumn{8}{|c|}{$\mathrm{HgF} 2$} \\
\hline $\mathrm{Hg}$ & 0.000000 & 0.000000 & 0.000000 & $\mathrm{Hg}$ & 0.000000 & 0.000000 & 0.000000 \\
\hline $\mathrm{F}$ & 0.000000 & 0.000000 & -1.912268 & $\mathrm{~F}$ & 0.000000 & 0.000000 & -1.901529 \\
\hline $\mathrm{F}$ & 0.000000 & 0.000000 & 1.912268 & $\mathrm{~F}$ & 0.000000 & 0.000000 & 1.901529 \\
\hline \multicolumn{8}{|c|}{$\mathrm{HgF} 4$} \\
\hline $\mathrm{Hg}$ & 0.000000 & 0.000000 & 0.000000 & $\mathrm{Hg}$ & 0.000000 & 0.000000 & 0.000000 \\
\hline $\mathrm{F}$ & 0.000000 & -1.883950 & 0.000000 & $\mathrm{~F}$ & 0.000000 & -1.872664 & 0.000000 \\
\hline $\mathrm{F}$ & 0.000000 & 1.883950 & 0.000000 & $\mathrm{~F}$ & 0.000000 & 1.872664 & 0.000000 \\
\hline $\mathrm{F}$ & -1.883950 & 0.000000 & 0.000000 & $\mathrm{~F}$ & -1.872664 & 0.000000 & 0.000000 \\
\hline $\mathrm{F}$ & 1.883950 & 0.000000 & 0.000000 & $\mathrm{~F}$ & 1.872664 & 0.000000 & 0.000000 \\
\hline \multicolumn{8}{|c|}{$\mathrm{HgF} 4 \quad(\mathrm{TS})$} \\
\hline $\mathrm{Hg}$ & 0.000000 & 0.000000 & 0.181728 & $\mathrm{Hg}$ & 0.000000 & 0.000000 & 0.192482 \\
\hline $\mathrm{F}$ & 0.000000 & -1.775220 & 1.025004 & $\mathrm{~F}$ & 0.000000 & -1.798707 & 0.949447 \\
\hline $\mathrm{F}$ & 0.000000 & 0.914184 & -1.832682 & $\mathrm{~F}$ & 0.000000 & 0.936060 & -1.804921 \\
\hline $\mathrm{F}$ & 0.000000 & 1.775220 & 1.025004 & $\mathrm{~F}$ & 0.000000 & 1.798707 & 0.949447 \\
\hline $\mathrm{F}$ & 0.000000 & -0.914184 & -1.832682 & $\mathrm{~F}$ & 0.000000 & -0.936060 & -1.804921 \\
\hline \multicolumn{8}{|c|}{$\mathrm{Cl2}$} \\
\hline $\mathrm{Cl}$ & 0.000000 & 0.000000 & -0.996200 & $\mathrm{Cl}$ & 0.000000 & 0.000000 & -0.996241 \\
\hline $\mathrm{Cl}$ & 0.000000 & 0.000000 & 0.996200 & $\mathrm{Cl}$ & 0.000000 & 0.000000 & 0.996241 \\
\hline \multicolumn{8}{|c|}{$\mathrm{HgCl} 2$} \\
\hline $\mathrm{Hg}$ & 0.000000 & 0.000000 & 0.000000 & $\mathrm{Hg}$ & 0.000000 & 0.000000 & 0.000000 \\
\hline $\mathrm{Cl}$ & 0.000000 & 0.000000 & -2.257751 & $\mathrm{Cl}$ & 0.000000 & 0.000000 & -2.254092 \\
\hline $\mathrm{Cl}$ & 0.000000 & 0.000000 & 2.257751 & $\mathrm{Cl}$ & 0.000000 & 0.000000 & 2.254092 \\
\hline \multicolumn{8}{|c|}{$\mathrm{HgCl} 4$} \\
\hline $\mathrm{Hg}$ & 0.000000 & 0.000000 & 0.000000 & $\mathrm{Hg}$ & 0.000000 & 0.000000 & 0.000000 \\
\hline $\mathrm{Cl}$ & 0.000000 & -2.303451 & 0.000000 & $\mathrm{Cl}$ & 0.000000 & -2.304490 & 0.000000 \\
\hline $\mathrm{Cl}$ & 0.000000 & 2.303451 & 0.000000 & $\mathrm{Cl}$ & 0.000000 & 2.304490 & 0.000000 \\
\hline $\mathrm{Cl}$ & -2.303451 & 0.000000 & 0.000000 & $\mathrm{Cl}$ & -2.304490 & 0.000000 & 0.000000 \\
\hline $\mathrm{Cl}$ & 2.303451 & 0.000000 & 0.000000 & $\mathrm{Cl}$ & 2.304490 & 0.000000 & 0.000000 \\
\hline \multicolumn{8}{|c|}{$\mathrm{HgCl} 4$ (TS) } \\
\hline $\mathrm{Hg}$ & 0.000000 & 0.000000 & 0.164451 & $\mathrm{Hg}$ & 0.000000 & 0.000000 & 0.162716 \\
\hline $\mathrm{Cl}$ & 0.000000 & -1.843076 & 1.610824 & $\mathrm{Cl}$ & 0.000000 & -1.841997 & 1.611893 \\
\hline $\mathrm{Cl}$ & 0.000000 & 1.231649 & -1.997769 & $\mathrm{Cl}$ & 0.000000 & 1.240765 & -1.994753 \\
\hline $\mathrm{Cl}$ & 0.000000 & 1.843076 & 1.610824 & $\mathrm{Cl}$ & 0.000000 & 1.841997 & 1.611893 \\
\hline $\mathrm{Cl}$ & 0.000000 & -1.231649 & -1.997769 & $\mathrm{Cl}$ & 0.000000 & -1.240765 & -1.994753 \\
\hline \multicolumn{8}{|c|}{$(\mathrm{CN}) 2$} \\
\hline $\mathrm{C}$ & 0.000000 & 0.000000 & -0.687711 & $\mathrm{C}$ & 0.000000 & 0.000000 & -0.687711 \\
\hline C & 0.000000 & 0.000000 & 0.687711 & $\mathrm{C}$ & 0.000000 & 0.000000 & 0.687711 \\
\hline $\mathrm{N}$ & 0.000000 & 0.000000 & -1.839155 & $\mathrm{~N}$ & 0.000000 & 0.000000 & -1.839155 \\
\hline $\mathrm{N}$ & 0.000000 & 0.000000 & 1.839155 & $\mathrm{~N}$ & 0.000000 & 0.000000 & 1.839155 \\
\hline \multicolumn{8}{|c|}{$\mathrm{Hg}(\mathrm{CN}) 2$} \\
\hline $\mathrm{Hg}$ & 0.000000 & 0.000000 & 0.000000 & $\mathrm{Hg}$ & 0.000000 & 0.000000 & 0.000000 \\
\hline $\mathrm{C}$ & 0.000000 & 0.000000 & -1.998581 & $\mathrm{C}$ & 0.000000 & 0.000000 & -1.952892 \\
\hline C & 0.000000 & 0.000000 & 1.998581 & $\mathrm{C}$ & 0.000000 & 0.000000 & 1.952892 \\
\hline $\mathrm{N}$ & 0.000000 & 0.000000 & -3.150775 & $\mathrm{~N}$ & 0.000000 & 0.000000 & -3.104423 \\
\hline $\mathrm{N}$ & 0.000000 & 0.000000 & 3.150775 & $\mathrm{~N}$ & 0.000000 & 0.000000 & 3.104423 \\
\hline \multicolumn{8}{|c|}{$\mathrm{Hg}(\mathrm{CN}) 4$} \\
\hline $\mathrm{Hg}$ & 0.000000 & 0.000000 & 0.000000 & $\mathrm{Hg}$ & 0.000000 & 0.000000 & 0.000000 \\
\hline $\mathrm{C}$ & 0.000000 & 2.010264 & 0.000000 & $\mathrm{C}$ & 0.000000 & 1.971387 & 0.000000 \\
\hline C & 0.000000 & -2.010264 & 0.000000 & $\mathrm{C}$ & 0.000000 & -1.971387 & 0.000000 \\
\hline $\mathrm{C}$ & 2.010264 & 0.000000 & 0.000000 & $\mathrm{C}$ & 1.971387 & 0.000000 & 0.000000 \\
\hline C & -2.010264 & 0.000000 & 0.000000 & $\mathrm{C}$ & -1.971387 & 0.000000 & 0.000000 \\
\hline $\mathrm{N}$ & 0.000000 & 3.160980 & 0.000000 & $\mathrm{~N}$ & 0.000000 & 3.121334 & 0.000000 \\
\hline $\mathrm{N}$ & 0.000000 & -3.160980 & 0.000000 & $\mathrm{~N}$ & 0.000000 & -3.121334 & 0.000000 \\
\hline $\mathrm{N}$ & 3.160980 & 0.000000 & 0.000000 & $\mathrm{~N}$ & 3.121334 & 0.000000 & 0.000000 \\
\hline $\mathrm{N}$ & -3.160980 & 0.000000 & 0.000000 & $\mathrm{~N}$ & -3.121334 & 0.000000 & 0.000000 \\
\hline
\end{tabular}




\begin{tabular}{|llrrlrrr|}
$\mathrm{Hg}(\mathrm{CN}) 4$ & (TS) & & & & & \\
$\mathrm{Hg}$ & 0.000000 & 0.000000 & 0.100517 & $\mathrm{Hg}$ & 0.000000 & 0.000000 & 0.099025 \\
$\mathrm{C}$ & 0.000000 & 0.957419 & -1.717180 & $\mathrm{C}$ & 0.000000 & 0.930037 & -1.699683 \\
$\mathrm{C}$ & 0.000000 & -0.957419 & -1.717180 & $\mathrm{C}$ & 0.000000 & -0.930037 & -1.699683 \\
$\mathrm{C}$ & 0.000000 & 1.527874 & 1.447661 & $\mathrm{C}$ & 0.000000 & 1.500572 & 1.425961 \\
$\mathrm{C}$ & 0.000000 & -1.527874 & 1.447661 & $\mathrm{C}$ & 0.000000 & -1.500572 & 1.425961 \\
$\mathrm{~N}$ & 0.000000 & 2.391645 & 2.209470 & $\mathrm{~N}$ & 0.000000 & 2.358502 & 2.193505 \\
$\mathrm{~N}$ & 0.000000 & -2.391645 & 2.209470 & $\mathrm{~N}$ & 0.000000 & -2.358502 & 2.193505 \\
$\mathrm{~N}$ & 0.000000 & 1.757026 & -2.552837 & $\mathrm{~N}$ & 0.000000 & 1.741021 & -2.524748 \\
$\mathrm{~N}$ & 0.000000 & -1.757026 & -2.552837 & $\mathrm{~N}$ & 0.000000 & -1.741021 & -2.524748 \\
\hline
\end{tabular}


Table S3: Harmonic vibrational frequencies (in $\mathrm{cm}^{-1}$ ) of $\mathrm{X}_{2}, \mathrm{HgX}_{2}$, and $\mathrm{HgX}_{4}(\mathrm{X}=\mathrm{F}, \mathrm{Cl}$, and $\mathrm{CN})$.

\begin{tabular}{|c|c|c|c|}
\hline Mol. & Vib. mode & sf-X2C & $\mathrm{X} 2 \mathrm{C}$ \\
\hline $\mathrm{F}_{2}$ & $1 \sigma_{g}^{+}$ & 1104 & 1104 \\
\hline \multirow{3}{*}{$\mathrm{HgF}_{2}$} & $1 \pi_{u}$ & 179 & 185 \\
\hline & $1 \sigma_{g}^{+}$ & 584 & 564 \\
\hline & $1 \sigma_{u}^{+}$ & 662 & 643 \\
\hline \multirow[t]{7}{*}{$\mathrm{HgF}_{4}$} & $1 b_{1 u}$ & 175 & 179 \\
\hline & $1 b_{1 g}$ & 224 & 230 \\
\hline & $1 a_{2 u}$ & 227 & 234 \\
\hline & $1 e_{u}$ & 251 & 257 \\
\hline & $1 b_{2 g}$ & 598 & 576 \\
\hline & $1 a_{1 g}$ & 603 & 586 \\
\hline & $2 e_{u}$ & 685 & 666 \\
\hline $\mathrm{Cl}_{2}$ & $1 \sigma_{g}^{+}$ & 576 & 576 \\
\hline \multirow[t]{3}{*}{$\mathrm{HgCl}_{2}$} & $1 \pi_{u}$ & 102 & 102 \\
\hline & $1 \sigma_{g}^{+}$ & 354 & 339 \\
\hline & $1 \sigma_{u}^{+}$ & 408 & 387 \\
\hline \multirow[t]{7}{*}{$\mathrm{HgCl}_{4}$} & $1 b_{1 u}$ & 71 & 67 \\
\hline & $1 a_{2 u}$ & 127 & 122 \\
\hline & $1 b_{1 g}$ & 145 & 145 \\
\hline & $1 e_{u}$ & 146 & 145 \\
\hline & $1 b_{2 g}$ & 307 & 293 \\
\hline & $1 a_{1 g}$ & 332 & 320 \\
\hline & $2 e_{u}$ & 378 & 360 \\
\hline \multirow{5}{*}{$(\mathrm{CN})_{2}$} & $1 \pi_{u}$ & 254 & 254 \\
\hline & $1 \pi_{g}$ & 557 & 557 \\
\hline & $1 \sigma_{g}^{+}$ & 892 & 892 \\
\hline & $1 \sigma_{u}^{+}$ & 2291 & 2291 \\
\hline & $2 \sigma_{g}^{+}$ & 2467 & 2467 \\
\hline \multirow[t]{7}{*}{$\mathrm{Hg}(\mathrm{CN})_{2}$} & $1 \pi_{u}^{\delta}$ & 80 & 86 \\
\hline & $1 \pi_{g}$ & 257 & 277 \\
\hline & $2 \pi_{u}$ & 369 & 399 \\
\hline & $1 \sigma_{g}^{+}$ & 444 & 446 \\
\hline & $1 \sigma_{u}^{+}$ & 475 & 474 \\
\hline & $2 \sigma_{u}^{+}$ & 2327 & 2336 \\
\hline & $2 \sigma_{g}^{+}$ & 2328 & 2337 \\
\hline \multirow[t]{16}{*}{$\mathrm{Hg}(\mathrm{CN})_{4}$} & $1 b_{1 u}$ & 70 & 74 \\
\hline & $1 b_{1 g}$ & 91 & 98 \\
\hline & $1 a_{2 u}$ & 91 & 97 \\
\hline & $1 e_{u}$ & 93 & 101 \\
\hline & $1 e_{g}$ & 254 & 271 \\
\hline & $1 a_{2 g}$ & 276 & 297 \\
\hline & $2 b_{1 u}$ & 365 & 386 \\
\hline & $2 b_{1 g}$ & 378 & 405 \\
\hline & $2 a_{2 u}$ & 382 & 408 \\
\hline & $2 e_{u}$ & 391 & 420 \\
\hline & $1 b_{2 g}$ & 432 & 429 \\
\hline & $1 a_{1 g}$ & 436 & 439 \\
\hline & $3 e_{u}$ & 472 & 468 \\
\hline & $4 e_{u}$ & 2334 & 2342 \\
\hline & $2 b_{2 g}$ & 2335 & 2343 \\
\hline & $2 a_{1 g}$ & 2340 & 2349 \\
\hline
\end{tabular}

